\title{
Checking the correct operation of main measuring instruments on the flight model and prototype of AIST small spacecraft
}

\author{
Andry Sedelnikov ${ }^{1}$, Ekaterina Khnyryova, ${ }^{2,}$, and Tatiana Ivashova $^{3}$ \\ ${ }^{1}$ Samara National Research University, Department of Space Engineering, 34 Moskovskoyeshosse, Samara, Russia \\ ${ }^{2}$ Samara National Research University, Department of Further Mathematics, 34 Moskovskoyeshosse, Samara, Russia \\ ${ }^{3}$ Samara National Research University, Faculty of Electronics and Instrument Engineering, 34Moskovskoyeshosse, Samara, Russia
}

\begin{abstract}
Flight model and prototype of AIST small spacecraft were launched in 2013 and 2014 respectively. They were used for carrying out various scientific researches, as well as testing the operation of AIST series small spacecrafts. They were operated in an uncontrolled flight and did not have a fullyfeatured orbital motion control system. However, it has been possible to reduce the angular velocity by magnetorquers. To obtain the control laws, the angular velocity of small spacecraft was estimated by means of magnetometer sensors. On the flight model of AIST small spacecraft, the angular velocity reduction mode was used twice. In the first case, the angular velocity was reduced, in the second case, it did not change significantly. On the prototype of AIST small spacecraft, the angular velocity reduction mode was used three times. In all three cases, a significant increase in the angular velocity of small spacecraft rotation was observed. The paper describes a test for checking the magnetometer sensors correct operation. Based on this test, conclusions about the measuring equipment correct operation are made. Ineffective control of the angular velocity of small spacecraft rotation is most likely due to failure to take account of impact of scientific and supporting equipment on the measuring instruments.
\end{abstract}

\section{Introduction}

Flight model and prototype of AIST small spacecraft were identical in design. However, during their operation, the magnetorquers showed different efficiency in solving the problem of reducing the angular velocity of small spacecraft rotation. In [1] it is noted that the prototype had a significant initial angular velocity when it fell-away. In all other aspects, the operating conditions of both small spacecrafts were the same. It is assumed that one of the reasons for the magnetorquers ineffective work is insufficient quality of raw data. The parameters estimation errors of the small spacecraft rotational motion led to the obtaining a non-optimal control law. One of the possible explanation for this situation is the incorrect operation of the measuring instruments. Such incorrect operation can be caused by the failure of the measuring instruments and by the impact of scientific and supporting equipment on the measuring instruments. The proposed test for checking the correct operation will allow to determine possible failures of measuring instruments. At testing, it is necessary to study in detail the impact of scientific and supporting equipment in all normal modes of its operation on the measuring instruments in order to answer the question about measuring data accuracy.

\section{Methods}

A series of investigations is devoted to the checking the correct operation of magnetic measuring instruments on the flight model and prototype of AIST small spacecraft [2-5]. In the paper we propose a test for checking the correct operation based on the following assumptions.

The average orbital value of the induction vector's modulus of Earth's magnetic field is constant.

The average value of the measuring error is approximately the same for all measurement sessions. Then the following limitations must be satisfied with an unlimited increase in the measurements number:

$$
\begin{gathered}
\lim _{n \rightarrow \infty}(|\overline{\vec{B}}|-|\hat{\overrightarrow{\vec{B}}}|)=0, \\
\lim _{n \rightarrow \infty} D|\hat{\overrightarrow{\vec{B}}}|=0
\end{gathered}
$$

where $|\overline{\vec{B}}|$ is the average orbital value of the induction vector's modulus of Earth's magnetic field; $|\hat{\vec{B}}|$ is sample average value of the induction modulus; $n$ is the measurements number; $D$ is variance.

\footnotetext{
* Corresponding author: khnyryova@gmail.com
} 


\subsection{Test for measuring instruments of the AIST small spacecraft flight model}

For the flight model of AIST small spacecraft it is typical a stable decrease in the average orbital value variance of the induction vector's modulus of Earth's magnetic field. However, the number of measurement sessions was insufficient to prove the correspondence between the average orbital value variance and the measuring error. There was much more measuring data for the prototype of the AIST small spacecraft, which allowed more detailed investigation the correct operation of measuring instruments.

In Table 1 the main data parameters are shown which were used to check the correct operation of measuring instruments on the flight model of AIST small spacecraft.

Table 1. The samples parameters of Earth's magnetic field measurement sessions on the AIST small spacecraft flight model.

\begin{tabular}{|c|c|c|c|c|}
\hline Date & $\begin{array}{c}\text { Sample } \\
\text { size }\end{array}$ & $\begin{array}{c}\text { Number of turns } \\
\text { per measure- } \\
\text { ment session }\end{array}$ & \multicolumn{2}{|c|}{ Total sample size } \\
\hline 27.04 .13 & 1150 & 1.2 & 1149 & 1149 \\
\hline 29.04 .13 & 400 & 0.6 & 1549 & 1548 \\
\hline 10.05 .13 & 1350 & 1.7 & 2899 & 2898 \\
\hline 14.05 .13 & 2350 & 2.6 & 5248 & 5246 \\
\hline 16.05 .13 & 1699 & 2.1 & 6726 & 6717 \\
\hline 20.05 .13 & 1750 & 2.3 & 8434 & 8426 \\
\hline
\end{tabular}

The dynamic pattern of the average orbital value of the induction vector's modulus of Earth's magnetic field and its variance are shown in Figures 1 and 2.

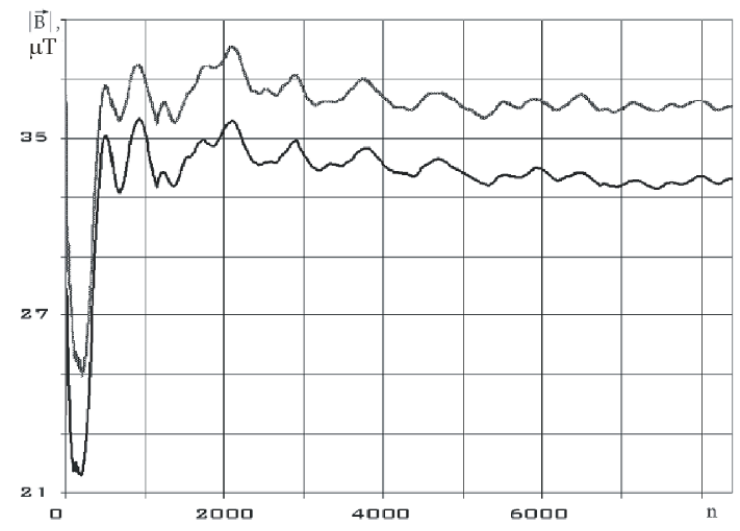

Fig. 1. The dynamic pattern of the average value of the total sample for sensor No. 1 (lower curve) and sensor No. 2 (upper curve).

Analysis of these dependences shows that the sample variance decreases with increasing sample size. The error of indirect measurements of the induction vector's modulus of Earth's magnetic field is $0.7 \mu T$ approximately. The dependencies presented in Fig. 2 do not allow to conclude that the variance of the average orbital value of the induction vector's modulus of Earth's magnetic field for all used measurements is explained by the declared measuring error of separate measuring instruments. Measuring error is $\pm 0.5 \mu T$ on the passport. If we assume that the indirect measurements error is approximately equal to the mean-square deviation, then in
Fig. 2 the variance of $0.5 \mu T^{2}$ can be explained by the measurement error. The minimum variance for sensor No. 2 by the total sample (Table 1) is $2.4 \mu T^{2}$ approximately. The variance estimate of the average orbital value is tenable, but it can be shifted on small samples. Therefore, the sample size of 8400 points does not allow us to state unambiguously that the variance in Fig. 2 is the measurement error.

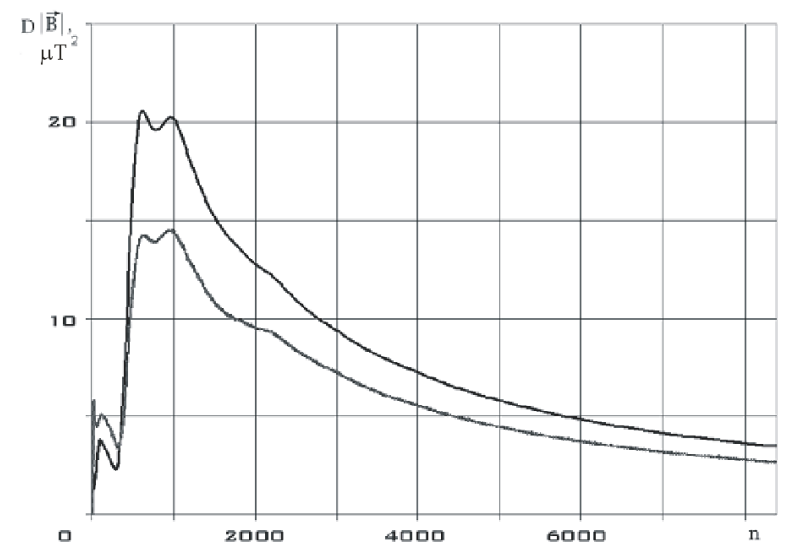

Fig. 2. The dynamic pattern of sampling variance of the total sample for sensor No. 1 (upper curve) and sensor No. 2 (lower curve).

\subsection{Test for measuring instruments of the AIST small spacecraft prototype}

For the AIST small spacecraft prototype, all the measurement data were divided into two types. The first type contained the orbital eclipse part of the small spacecraft, the second type included data obtained only in the orbital sunny part of the small spacecraft. The measurements from sunny part were used to check the correct operation of main measuring instruments. The electrical battery was always charged and it can be concluded that the supporting equipment had a uniform effect on the measuring instruments. In the case of both sunny and eclipse parts of the orbit, that effect would be non-uniform due to the lack of charging current in the eclipse part of the orbit.

It is also necessary to take into account the feature of the measurement channel X1 of the sensor No. 1, noted in [3]. The investigations presented in this paper do not allow us to recognize measurements as abnormal. However, in almost all normal modes of operation, the average value for the corresponding component of the magnetic induction vector was the last in the ranked series of average values of six measurement channels. In addition, the variance was the minimum for all channels. None of the three criteria for checking abnormal values used in [4] made it impossible to recognize the average value and variance of the channel X1 as the outlier. Such feature on a small part of the measurements could be explained by the low angular velocity of small spacecraft rotation [5]. As a result, the components of the induction vector's modulus of Earth's magnetic field in the instrumental bound coordinate system would change insignificantly. However, in [1] was noted that the angular velocity of the AIST small spacecraft prototype rotational 
motion was higher than the angular velocity of the AIST small spacecraft flight model rotational motion due to giving it the initial angular velocity. Measurements were made over a long period.

In Table 2 the main measuring data parameters are shown for the AIST small spacecraft prototype.

Table 2. The samples parameters of Earth's magnetic field measurement sessions on the AIST small spacecraft prototype.

\begin{tabular}{|c|c|c|c|c|}
\hline & Date & $\begin{array}{c}\text { Sample } \\
\text { size }\end{array}$ & $\begin{array}{c}\text { Number of } \\
\text { turns per } \\
\text { measurement } \\
\text { session }\end{array}$ & \multicolumn{2}{|c|}{$\begin{array}{c}\text { Total sample } \\
\text { size }\end{array}$} \\
\hline 11.01 .14 & 1850 & 2.0 & 1848 & 1849 \\
\hline 14.01 .14 & 1704 & 1.9 & 3550 & 3552 \\
\hline 17.01 .14 & 1459 & 1.8 & 5004 & 5007 \\
\hline 24.01 .14 & 1500 & 1.8 & 6500 & 6506 \\
\hline 28.01 .14 & 3328 & 3.7 & 9825 & 9830 \\
\hline 31.01 .14 & 2000 & 2.4 & 11820 & 11828 \\
\hline 07.02 .14 & 3449 & 3.7 & 15265 & 15274 \\
\hline 11.02 .14 & 1850 & 2.0 & 17111 & 17122 \\
\hline 15.02 .14 & 392 & 0.3 & 17499 & 17509 \\
\hline 19.03 .14 & 1863 & 2.7 & 19360 & 19372 \\
\hline 25.03 .14 & 2187 & 2.8 & 21543 & 21563 \\
\hline 28.03 .14 & 1331 & 2.7 & 22843 & 22860 \\
\hline 07.04 .14 & 1561 & 2.3 & 24401 & 24422 \\
\hline 27.06 .14 & 3115 & 4.0 & 27513 & 27538 \\
\hline 18.07 .14 & 2789 & 3.9 & 30297 & 30326 \\
\hline 23.07 .14 & 2241 & 4.2 & 32519 & 32546 \\
\hline 25.07 .14 & 3005 & 4.0 & 35520 & 35553 \\
\hline 01.08 .14 & 2725 & 4.0 & 38240 & 38274 \\
\hline 05.08 .14 & 2643 & 4.0 & 40880 & 40915 \\
\hline 12.08 .14 & 2617 & 3.9 & 43490 & 43522 \\
\hline 15.08 .14 & 3141 & 4.0 & 46626 & 46666 \\
\hline 21.08 .14 & 2321 & 2.9 & 48943 & 48985 \\
\hline 14.10 .14 & 3425 & 4.0 & 52358 & 52400 \\
\hline & & & & \\
\hline
\end{tabular}

The dynamic pattern of the average orbital value of the induction vector's modulus of Earth's magnetic field and its variance are shown in Figures 3 and 4.

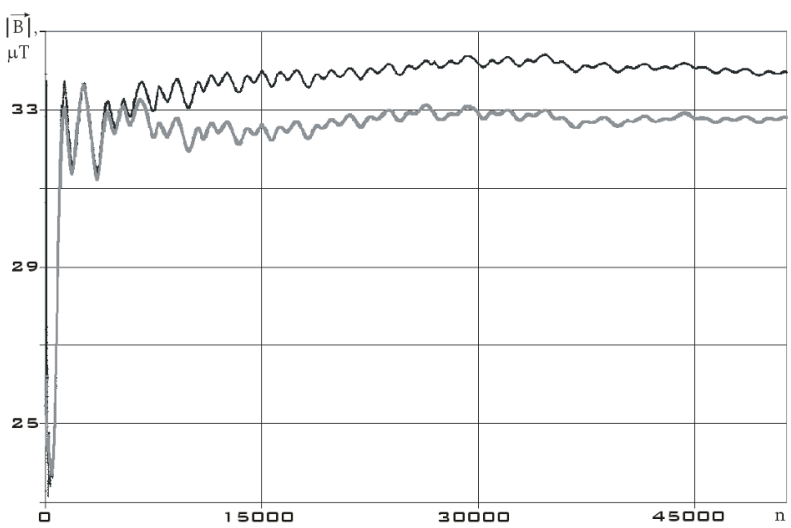

Fig. 3. The dynamic pattern of the average value of the total sample for sensor No. 1 (upper curve) and sensor No. 2 (lower curve).

Measuring instruments on the flight model and prototype of AIST small spacecraft were identical. And the error of indirect measurements of the Earth's magnetic field induction vector's modulus is also $0.7 \mu T$. Unlike Fig. 2, in Fig. 4, the variance minimum value for sensor
No. 2 is about $0.4 \mu T^{2}$. As measurement error we can still explain the variance of $0.5 \mu T^{2}$. Consequently, a significant increase in the sample size from 8,400 to 52,400 measurements changed the situation. The presented results allow us to make a conclusion about fault-free performance of the sensor No. 2. To investigate the effect on the sensor of scientific and supporting equipment it is necessary to test the separate vector components, in addition to the integral test for the induction vector's modulus of Earth's magnetic field.

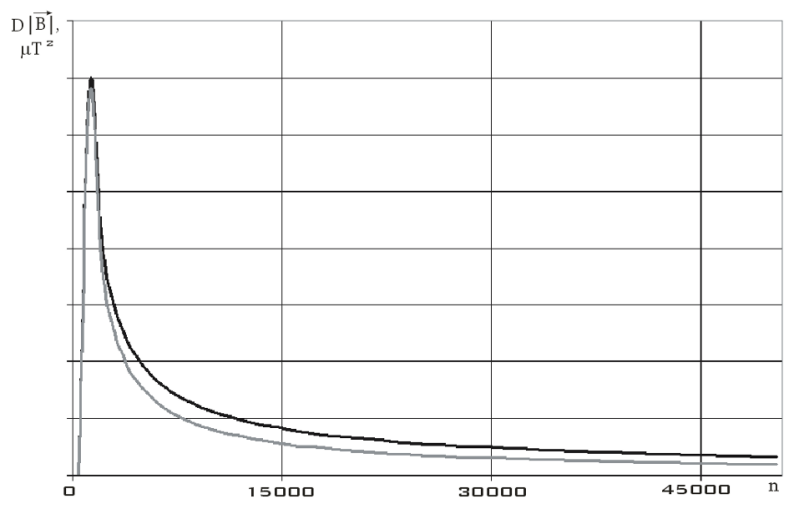

Fig. 4. The dynamic pattern of sampling variance of the total sample for sensor No. 1 (upper curve) and sensor No. 2 (lower curve).

\section{Results and discussion}

The variance of the sensor No. 1 is about $0.7 \mu T^{2}$ and cannot be explained completely by the measurement error. The reason for the difference in the two sensors variances can be the features of the X1 measurement channel operation. We already said about it and now we can make several assumptions:

1. Failure in calibration of the channel X1. Perhaps after installing the sensors in the small spacecraft internal environment, an abnormal situation arose, and as a result the channel calibration changed. The consistency of the related channels X1 of sensor No. 1 and Y2 of sensor No. 2 is the worst;

2. The scientific and supporting equipment design was made in such a way that the maximum effect was on the channel X1 of the sensor No. 1. As a result, measurements on this channel were most significantly distorted by the magnetic fields of the elements and devices of the small spacecraft.

The magnetorquers operated in the microacceleration compensation mode to reduce the angular velocity module of the small spacecraft rotation. The second assumption is supported by the fact that in the microacceleration compensation mode, the average values and variances of the corresponding component of the induction vector's modulus of Earth's magnetic field were not the last ones in any of the three cases of using this mode. It can be explained by the fact that the magnetic remanence of the magnetorquers reduced the influence on the channel X1 of the sensor No. 1 other scientific and supporting equipment. 
Thus, based on the results of the investigation, we can state that:

1. Failures of the standard measuring instruments of the Earth's magnetic field induction vector's components on the flight model and prototype of AIST small spacecraft were not detected during the testing of correct operation.

2. For the flight model of AIST small spacecraft the number of measurements were insufficient to explain the variance of the average orbital value of the Earth's magnetic field induction vector by the measurement error.

3 . In both cases, the variance of sensor No. 1 was greater than the variance of sensor No. 2. It can be explained by the similarity in design of two small spacecrafts, which determine the same impact degree of the scientific and supporting equipment on the measuring instruments.

4. In the analysis of variances, the hypothesis [4] about the features of the measuring channel X1 of the sensor No. 1 was confirmed.

5. The hypotheses $[1,6]$ were confirmed. The data from the sensor No. 2 on both small spacecrafts allows to reconstruct more accurately the rotational motion of these small spacecrafts.

6. To investigate the impact of scientific and supporting equipment on the correct operation of measuring instruments, it is necessary to test the channels. At the same time, it is necessary to give a physical explanation to the value to which the average sample values of the separate measurement channels will tend with an unlimited increase in the sample.

This work is supported by the Ministry of Education and Science of the Russian Federation in the framework of the State Assignments to higher education institutions and research organizations in the field of scientific activity (the project \# 9.1616.2017/ПЧ).

\section{References}

1. V. Abrashkin, Yu. Puzin, A. Filippov, K. Voronov, A. Piyakov, N. Semkin, V. Sazonov, S. Chebukov, Uncontrolled rotational motion of the AIST small spacecraft prototype, Cosmic Research, 55(2), 128141 (2017)

2. M. Belyaev, O. Volkov, M. Monakhov, V. Sazonov, Estimating the accuracy of the technique of reconstructing the rotational motion of a satellite based on the measurements of its angular velocity and the magnetic field of the Earth, Cosmic Research, 55 (5), 345-360 (2017)

3. A. Sedelnikov, Fast analysis of onboard measurements of the Earth magnetic field for the purpose of microaccelerations decrement on board of the "AIST" small spacecraft during its uncontrolled orbital flight, International Review of Aerospace Engineering, 11(2), 76-83 (2018)

4. A. Sedelnikov, Mean of microaccelerations estimate in the small spacecraft internal environment with the use of fuzzy sets, Microgravity Science and Technology, Articleinpress (2018)
5. A. Sedelnikov, Modeling of microaccelerations caused by running of attitude-control engines of spacecraft with elastic structural elements, Microgravity Science and Technology, 28 (5), 491498 (2016)

6. V. Abrashkin, Yu. Puzin, A. Filippov, K. Voronov, A. Piyakov, N. Semkin, V. Sazonov, S. Chebukov, Uncontrolled attitude motion of the small satellite AIST. Cosmic Research, 53 (5), 360-373 (2015) 